\title{
Calciphylaxis: Early Detection and Off-Label Treatment With Sodium Thiosulfate
}

\author{
Nicholas London ${ }^{\mathrm{a}}$, Ahmad Eter ${ }^{\mathrm{b}, \mathrm{c}}$
}

\begin{abstract}
Calciphylaxis or calcific uremic arteriolopathy (CUA) is a rare disorder of calcium and phosphate metabolism most often manifesting in end-stage renal disease (ESRD) patients. The typical clinical picture is that of a necrotic cutaneous ulceration with focal or diffuse distribution, most often manifesting on the lower extremities. We report a 49-year-old Caucasian male with ESRD on hemodialysis who presented to the emergency department with complaints of lower extremity pain and multiple cutaneous necrotic lesions on his lower extremities. Patient reports seeking assistance from several medical providers over a 3-month period. Upon admission and further evaluation, a diagnosis of calciphylaxis was made clinically and confirmed by a skin biopsy. Sodium thiosulfate was initiated and the clinical picture improved significantly. Sodium thiosulfate is traditionally used as an antidote for cyanide poisoning, and it has demonstrated beneficial outcomes in most patients afflicted with CUA. The rationale behind sodium thiosulfate therapy in CUA is its role in chelating calcium into calcium thiosulfate, which increases its solubility leading to improved renal clearance. It is essential to raise awareness amongst physicians and medical practitioners alike, as early recognition and initiation of appropriate treatment can improve the patients' quality of life and more importantly, decrease mortality.
\end{abstract}

Keywords: Calciphylaxis; Calcific uremic arteriolopathy; End-stage renal disease; CKD; Sodium thiosulfate; Necrosis; Biopsy

\section{Introduction}

Sodium thiosulfate therapy in the management of calciphylaxis is a relatively new approach. Although currently unapproved by the US Food and Drug Administration for treatment in calciphylaxis, sodium thiosulfate shows promising results

Manuscript submitted April 26, 2017, accepted May 18, 2017

aAvalon University School of Medicine, PO Box 480, Girard, OH 44420, USA bDivision of Nephrology, Department of Medicine, Beckley Appalachian Regional Healthcare Hospital, 306 Stanaford Rd, Beckley, WV 2580, USA

${ }^{\mathrm{c} C}$ Corresponding Author: Ahmad Eter, Division of Nephrology, Department of Medicine, Beckley Appalachian Regional Healthcare Hospital, 306 Stanaford Rd, Beckley, WV 2580, USA. Email: Ahmadeter1@hotmail.com

doi: https://doi.org/10.14740/wjnu298e compared to more traditional therapies, which include low calcium dialysates in hemodialysis, selective vitamin D analogs, cinacalcet and wound care [1]. Early identification and rapid initiation of treatment is of the utmost importance due to the high mortality risk associated with infection and possible sepsis. A significant improvement upon administration of the correct treatment regimen was clearly evident in this case. Other studies have also demonstrated more favorable outcomes in patients treated with sodium thiosulfate.

\section{Background}

The pathophysiology of calciphylaxis continues to be poorly understood. A two-stage process has been hypothesized. The first stage includes vascular injury, characterized by mural calcification, intimal hyperplasia and endovascular fibrosis. In the second stage, further vascular damage is elicited by local trauma, hypotension or thrombosis. The proposed mechanism leads to development of an ischemic infarct, dystrophic calcification or further ulceration [2]. Calcification often involves the medial layer of arterioles; however, involvement of the intima and interstitium of adipose tissue has been described [3]. Satisfactory treatment remains challenging due to high infection risk and mortality rate with an estimated 1-year survival of $45.8 \%$ [4]. Early recognition and administration of sodium thiosulfate may decrease the progression of skin necrosis resulting in improved outcomes, as ulceration carries a mortality of greater than $80 \%$ as a result of local and systemic infections and sepsis [5]. Unfortunately, early recognition is not always possible due to the relative obscurity of this uncommon disorder. With a necrotic appearance mimicking other pathological disorders, early recognition can prove to a diagnostic challenge.

\section{Case Report}

A 49-year-old Caucasian male with ESRD on hemodialysis presented to the emergency department with complaints of lower extremity pain and non-healing ulcers. Over a 3-month period, he presented to multiple medical providers complaining of lower extremity ulcers, which would not heal and were causing him debilitating pain. The patient reported being unable to walk more than a few steps without intense pain. The patient was previously diagnosed with peripheral vascular dis- 
Table 1. Patients' Labs Pertinent to the Current Condition

\begin{tabular}{llllll}
\hline Phosphorus & Albumin & Calcium & Corrected Ca & PTH & Ca $\times$ P \\
\hline $9.3 \mathrm{mg} / \mathrm{dL} \uparrow$ & $3.0 \mathrm{mg} / \mathrm{dL} \downarrow$ & $8.4 \mathrm{mg} / \mathrm{dL}$ & $9.2 \mathrm{mg} / \mathrm{dL}$ & $408.1 \mathrm{pg} / \mathrm{mL} \uparrow$ & $85.56 \uparrow$ \\
\hline
\end{tabular}

ease and lower extremity cellulitis, which upon further clinical and laboratory evaluation (Table 1), a skin biopsy was performed and it showed typical vascular changes of calciphylaxis (Fig. 1). The patient was administered sodium thiosulfate upon diagnosis and in the following weeks, displayed a marked improvement. The patient reported being able to bear weight on his lower extremity without excruciating pain and in addition, the ulcers displayed signs of healing with less induration and inflammation on the borders with continued treatment (Fig. 2). During admission, the patients' laboratory values were closely monitored and wound management was consistently consulted.

\section{Discussion}

Calciphylaxis most commonly occurs in patients with ESRD on dialysis, but may also occur in patients not undergoing dialysis. Painful lesions in the form of non-healing ulcers, subcutaneous nodules or necrosis should arouse immediate suspicion. Laboratory clues may or not be evident but are usually demonstrated as an increased parathyroid hormone (PTH) level, as well as elevations in calcium, phosphorus, and the calcium $\times$ phosphorus product. An additional risk factor such as concurrent warfarin use should be taken note of and any observations of elevations in the calcium $\times$ phosphorus product and/or PTH levels over past months may support the diagnosis.

However, it is very important to mention that although we have many ESRD patients on dialysis, the prevalence of calcific uremic arteriolopathy (CUA) is rare with a multifactorial etiology not completely understood currently and requires more than one risk factor (Table 2 [4-12]).

\section{Diagnosis}

The current gold standard for definitive diagnosis of suspected calciphylaxis is via biopsy. Often times biopsy is not performed due to coexisting risk factors such as active infection or septicemia, whereby creating a potential wound could exacerbate current infections or increase the prospect of superinfection and introduction of new dermal ulcerations. Due to the painful nature of the procedure, patients will on occasion refuse biopsy.

In situations where biopsy is not suitable, several non-invasive imaging studies are available, which although lacking in the conclusive nature of a biopsy, have demonstrated desirable results in certain cases. Using imagining techniques, evident calcium deposition in tissues where its presence is not characteristic, has been demonstrated by high-resolution computed tomography (CT), bone scans, plain radiographs and even mammography. Although useful, imaging techniques should not be used as a definitive means of diagnosing calciphylaxis. If a biopsy is not viable, and a strong clinical suspicion is present, initiation of treatment for suspected calciphylaxis may be beneficial, as the therapeutic benefit of sodium thiosulfate is distinctly evident early on.

As prior mentioned, early recognition of this obscure disorder is very important in selecting an appropriate treatment regimen which will ultimately decrease mortality rates and improve quality of life as well as adequately manage pain levels in afflicted patients. Due to the multifactorial nature of calci-

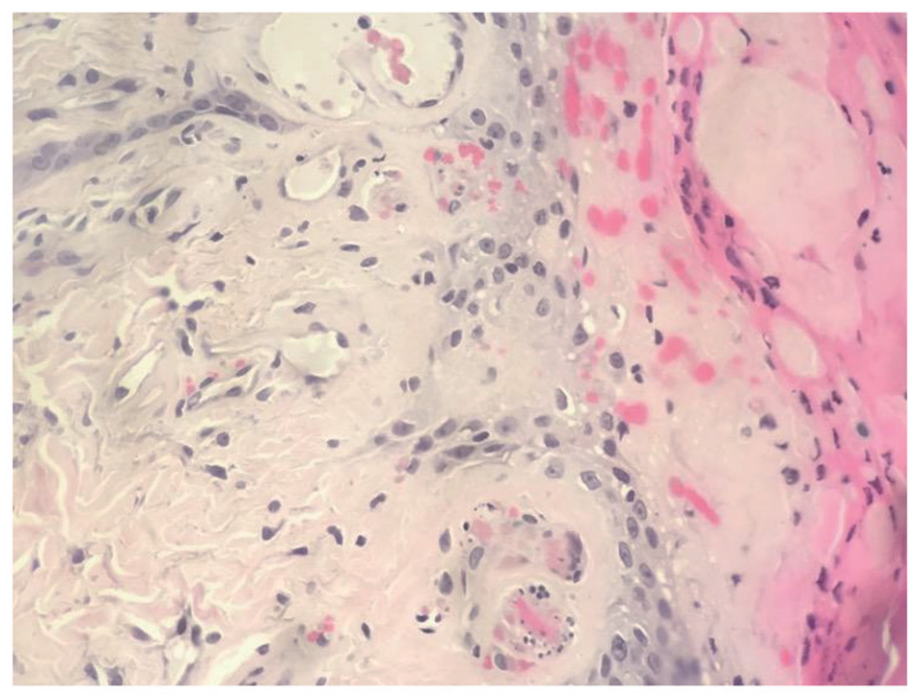

Figure 1. Biopsy findings. The histological section demonstrates fibrin thrombi within small blood vessels and ischemic necrosis of the epidermis, consistent with calcific uremic arteriolopathy (CUA). 

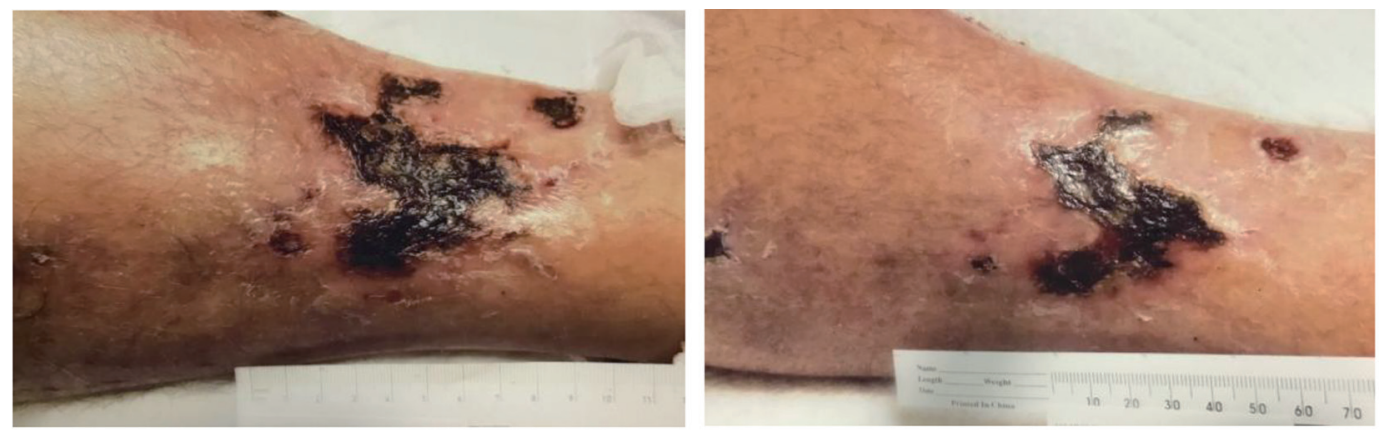

Figure 2. Necrotic ulcers before and after sodium thiosulfate.

Table 2. Risk Factors for CUA [4-12]

\begin{tabular}{ll}
\hline Risk factor & After reviewing many case reports, case series, and observational studies, the following were observed \\
\hline Age & CUA is more reported in patients in the fifth decade of life. \\
Sex & CUA is seen more in women 2:1 men [4]. \\
Race & CUA affected more whites compared to non-whites [4]. \\
$\begin{array}{l}\text { Calcium-phosphorus } \\
\text { metabolism }\end{array}$ & CUA is related to impaired calcium-phosphorus metabolism in dialysis patients as calcium, phosphorus, vitamin D, \\
Comorbidities & PTH levels and treatment used as vitamin D [7, 8]. \\
& CUA was observed with the presence of other medical diseases (DM-2, obesity, autoimmune conditions, \\
hedications & CUA wascoagulable conditions, and liver disease) [4, 5, 9-11]. \\
& $\begin{array}{l}\text { warfarin, corticosteroids, iron therapy, and trauma related to subcutaneous insulin or heparin injections have been } \\
\text { associated with increased calciphylaxis risk [12]. }\end{array}$ \\
\hline
\end{tabular}

phylaxis, in addition to sodium thiosulfate therapy, certain patients may benefit from concomitant parathyroidectomy with correction of underlying co-morbidities.

\section{Treatment}

A pharmacological cure for calciphylaxis has not been determined but a multi-interventional strategy which includes the use of sodium thiosulfate as the cornerstone has demonstrated promising results compared to the traditional therapeutic modalities which have thus far proved to be underwhelming [13].

\section{Prognosis}

CUA is a lethal disease and has significant morbidity and mortality. The response to any therapeutic regimen, however, appears to be poor. Mortality rates in long-term hemodialysis patients with CUA were almost three times higher than for long-term hemodialysis patients without CUA in the United States Renal Data System [14].

\section{Conclusion}

The off-label use of sodium thiosulfate is a promising advancement in the treatment of calciphylaxis. Although the disorder itself remains poorly understood, definitive treatment goals outweigh traditional management protocols such as treating the underlying symptoms. Further case studies leading to increased exposure of this infrequent disease process are important for providing an early diagnosis and initiation of appropriate treatment, thereby lowering mortality.

\section{References}

1. Generali, Joyce A., RPh, MS, FASHP (Editor) and Cada, Dennis J., PharmD, FASHP, FASCP*. Off-Label Drug Uses Sodium Thiosulfate: Calciphylaxis. Off-Label Drug Facts, Wolters Kluwer Health.

2. Yu Z, Gu L, Pang H, Fang Y, Yan H, Fang W. Sodium thiosulfate: an emerging treatment for calciphylaxis in dialysis patients. Case Rep Nephrol Dial. 2015;5(1):77-82.

3. Dauden E, Onate MJ. Calciphylaxis. Dermatol Clin. 2008;26(4):557-568, ix.

4. Weenig RH, Sewell LD, Davis MD, McCarthy JT, Pittelkow MR. Calciphylaxis: natural history, risk factor analysis, and outcome. J Am Acad Dermatol. 2007;56(4):569-579.

5. Fine A, Zacharias J. Calciphylaxis is usually non-ulcerating: risk factors, outcome and therapy. Kidney Int. 2002;61(6):2210-2217.

6. London N, Eter A. Calciphylaxis: Early detection and Off-label treatment with Sodium Thiosulfate. Table 2: 
Risk factors for CUA, p. 6, 2017.

7. Llach F. Calcific uremic arteriolopathy (calciphylaxis): an evolving entity? Am J Kidney Dis. 1998;32(3):514-518.

8. Nigwekar SU, Tamez H, Thadhani RI. Vitamin D and chronic kidney disease-mineral bone disease (CKDMBD). Bonekey Rep. 2014;3:498.

9. Bleyer AJ, Choi M, Igwemezie B, de la Torre E, White WL. A case control study of proximal calciphylaxis. Am J Kidney Dis. 1998;32(3):376-383.

10. Lee JL, Naguwa SM, Cheema G, Gershwin ME. Recognizing calcific uremic arteriolopathy in autoimmune disease: an emerging mimicker of vasculitis. Autoimmun Rev. 2008;7(8):638-643.

11. Harris RJ, Cropley TG. Possible role of hypercoagulabil- ity in calciphylaxis: review of the literature. J Am Acad Dermatol. 2011;64(2):405-412.

12. Nigwekar SU, Kroshinsky D, Nazarian RM, Goverman J, Malhotra R, Jackson VA, Kamdar MM, et al. Calciphylaxis: risk factors, diagnosis, and treatment. Am J Kidney Dis. 2015;66(1):133-146.

13. Nigwekar SU, Brunelli SM, Meade D, Wang W, Hymes J, Lacson E, Jr. Sodium thiosulfate therapy for calcific uremic arteriolopathy. Clin J Am Soc Nephrol. 2013;8(7):1162-1170.

14. Nigwekar SU, Solid CA, Ankers E, Malhotra R, Eggert W, Turchin A, Thadhani RI, et al. Quantifying a rare disease in administrative data: the example of calciphylaxis. J Gen Intern Med. 2014;29(Suppl 3):S724-731. 